\title{
RESET
}

Recherches en sciences sociales sur Internet

$10 \mid 2021$

Savoirs incertains

\section{Aux frontières du fact-checking}

Le « complotisme » comme révélateur des normes et des logiques d'un nouveau genre journalistique

Borderline fact-checking. How "conspiracy theories" expose the norms and logics of a new journalistic genre

\section{Elsa Jaubert et Vassili Rivron}

\section{(2) OpenEdition}

\section{Journals}

Édition électronique

URL : https://journals.openedition.org/reset/3173

DOI : 10.4000/reset.3173

ISSN : 2264-6221

Éditeur

Association Recherches en sciences sociales sur Internet

Référence électronique

Elsa Jaubert et Vassili Rivron, «Aux frontières du fact-checking », RESET [En ligne], 10 | 2021, mis en ligne le 13 mai 2021, consulté le 22 mai 2021. URL : http://journals.openedition.org/reset/3173 ; DOI : https://doi.org/10.4000/reset.3173

Ce document a été généré automatiquement le 22 mai 2021.

(c) Association Recherches en sciences sociales sur Internet 


\section{Aux frontières $\mathrm{du}$ fact-checking}

Le « complotisme » comme révélateur des normes et des logiques d'un nouveau genre journalistique

Borderline fact-checking. How "conspiracy theories" expose the norms and

logics of a new journalistic genre

Elsa Jaubert et Vassili Rivron

\section{Introduction}

1 Les «théories du complot » sont caractérisées par Michael Barkun (2015) comme une «connaissance stigmatisée» qui ne parvient pas à obtenir d'approbation institutionnelle, ou s'élabore indépendamment de celle-ci, et constitue un ensemble de visions déviantes par rapport à une parole autorisée. Cette vision quelque peu binaire («mainstream» / «déviant») attire cependant l'attention sur la dimension relationnelle de la construction des catégories de «journalisme» et de " complotisme ». Les énoncés dits complotistes prendraient ainsi place dans le champ de la controverse publique et de la confrontation des savoirs (Lemieux, 2007), où se déploient des dynamiques d'inclusion/exclusion et de hiérarchisation des messages.

2 Pourquoi parler d'un nouveau genre journalistique - le fact-checking - dans un dossier sur le complotisme ? Car, au-delà d'un phénomène en soi difficile à cerner dans son unité, le complotisme fait aussi l'objet de constructions externes, par des mécanismes de mise à l'écart, de « labellisation exogène infamante » (Taïeb, 2010 ; Giry, 2017), dans lesquels les journalistes interviendraient en tant qu' «entrepreneurs de morale » (Becker, 1963) contribuant à produire cette catégorie. Interroger les «fact-ckeckeurs " d'organes centraux de la presse quotidienne nationale sur la catégorie et leur rapport aux «théories du complot » permet d'évaluer dans quelle mesure ils s'inscrivent dans la controverse et s'ils alimentent ou non les mécanismes de stigmatisation (Goffman, 1975) mettant à l'index ou disqualifiant certains énoncés, leurs producteurs ou leurs relais de diffusion. Cela permet aussi d'appréhender les normes de ces nouvelles instances de construction d'une réalité factuelle partagée que sont les cellules de fact- 
checking. C'est également une porte d'entrée pour analyser le rôle de ces dernières dans les stratégies des organes de presse pour réaffirmer leur légitimité dans une démocratie représentative et reconquérir un public qui tend à être capté par d'autres acteurs et pratiques médiatiques.

Le fact-checking tel qu'il est mis en œuvre depuis les années 2000 aux États-Unis, puis en France à partir de 2008, a été dans un premier temps consacré à la vérification de la parole politique (Bigot, 2017a). Face à l'émergence de nouveaux circuits de l'information (Cardon, 2019) et à la " grande peur » que la propagation des "fake news » suscite dans l'opinion publique et chez les responsables politiques (Huygue, 2018; Koukoutsaki-Monnier, 2018), la pratique évolue et s'oriente depuis 2016 vers la vérification de tout type d'information qui circule et atteint une audience, quel qu'en soit l'émetteur et le média.

4 Cette enquête ${ }^{1}$ a été menée auprès d'organes à la fois emblématiques du rôle de la presse dans la formation de l'espace public et disposant d'un service de fact-checking agréé par l'International Fact-Checking Network $\left(\mathrm{IFCN}^{2}\right)$ qui sont au nombre de sept en France ${ }^{3}$. Une agence de presse (AFP), un quotidien national généraliste (Libération) et un journal gratuit (20 minutes) ont rapidement répondu à nos sollicitations ${ }^{4}$. Dix entretiens individuels semi-directifs, d'une durée de 30 minutes à plus d'une heure, ont été réalisés à Paris les 1er et 2 octobre 2019, dans les départements dédiés de trois rédactions: "CheckNews" à Libération (cinq entretiens), «Factuel» à l'AFP (trois entretiens) et « Fake Off » à 20 Minutes (deux entretiens). Ceux-ci portaient à la fois sur le rapport des journalistes aux catégories "théories du complot ", " complotisme » et " conspirationnisme », et sur leurs pratiques d'enquête et d'écriture. Une attention particulière a été accordée - lors des entretiens et de l'observation au sein des rédactions - aux outils techniques dont ils disposaient, liés pour certains aux contrats avec les plateformes sociales. Les trois cellules étudiées ont en effet en commun de participer au programme Facebook de lutte contre les fausses informations, mis en place en France en novembre $2017^{5}$. Cette étude s'appuie enfin sur l'analyse des articles produits et diffusés par ces cellules de fact-checking, consultables depuis un poste informatique standard au moment de l'enquête de terrain, donc tels qu'ils peuvent apparaître au public sur les sites des organes de presse ou sur les réseaux sociaux, afin d'examiner les usages de la catégorie "complotiste» dans ce type de production journalistique.

5 CheckNews est la cellule la plus importante en termes d'effectifs : huit personnes y travaillent à temps plein et une neuvième à mi-temps. Elle est issue de la rubrique Désintox, premier acteur du fact-checking en France, créé en 2008 à Libération. Sa spécificité aujourd'hui est de ne jamais s'auto-saisir, mais de répondre exclusivement aux questions posées directement par le public, ou aux signalements effectués auprès de Facebook. Les effectifs de Factuel à l'AFP sont plus variables : six à sept personnes s'y consacrent essentiellement au fact-checking, mais pas à temps complet. Leur modèle est fait de veille personnelle pour identifier les sujets intéressants à vérifier et de réponses à des sollicitations du public ou via l'outil Facebook. À 20 Minutes, deux journalistes travaillent à temps complet pour la rubrique Fake Off, avec le même fonctionnement que Factuel. Les dix journalistes spécialisés que nous avons rencontrés font partie d'un groupe qui compte actuellement au maximum une cinquantaine de professionnels en France (Vauchez, 2019). Les enquêtés avaient entre vingt-cinq et quarante-cinq ans, tous diplômés d'écoles de journalisme françaises. Ils ont tous déjà travaillé dans 
d'autres services de presse quotidienne ou de radio nationale. La position relative de chacun de ces agents (trajectoire et position professionnelle) dans les cellules, les organes de presse étudiés et le champ journalistique est cependant mise de côté pour nous focaliser ici sur les règles, logiques et représentations communes à ce corps professionnel à la fois nouveau et particulier.

6 L'objectif de cette contribution n'est pas d'aborder frontalement les ressorts $d u$ «complotisme» et des «théories du complot», ni de discuter des approches, de la définition et de la pertinence scientifique de ces termes (Giry, 2017 ; Butter \& Knight, 2015). Nous avons pour but de préciser la façon dont les discours et pratiques des journalistes fact-checkeurs contribuent en creux à définir les "théories du complot " comme déviances informationnelles. Alors que, dans le discours médiatique, "fake news » et "complotisme» sont fréquemment associés, et que les cellules de factchecking sont présentées comme des organes de lutte contre les premières, on aurait pu s'attendre à ce que le second ait une place plus saillante dans les produits et discours journalistiques résultant du processus de vérification. Or la réalité est plus nuancée.

7 L'analyse de contenu des entretiens menés montre comment les frontières sont tracées, établissant une séparation nette entre les faits " vérifiables » et des énoncés de nature " complotiste ", factuellement insaisissables. Le rôle du fact-checking est, selon celles et ceux qui le pratiquent, de donner à voir la construction de la factualité journalistique qui consiste en un recours méthodique aux formes instituées d'autorité et, plus subtilement, en la stigmatisation d'instances moins autorisées de production d'énoncés médiatiques. Cette nouvelle forme journalistique, qui met en scène ses principes de transparence et sa mission pédagogique pour regagner la confiance du public et redonner de la valeur à l'information produite, permet aux acteurs de réaffirmer clairement les principes de légitimation fonctionnelle du journalisme (Ruellan, 1992) dans les démocraties représentatives (Bougnoux, 2007). Par légitimation ou rhétorique fonctionnelle, nous entendrons dans cet article, la référence au journalisme comme rouage essentiel d'un espace public, répondant à la fois au droit des citoyens à une information indépendante (du pouvoir en place) permettant l'exercice éclairé de leurs devoirs démocratiques et à la nécessité d'un contre-pouvoir (le "quatrième pouvoir ») contrôlant l'activité de l'État et des élus entre deux scrutins. Le lien de confiance c'est-à-dire un rapport de légitimité au journalisme qui exerce une autorité qui lui est reconnue - repose à la fois sur la réaffirmation d'un idéal d'objectivité (réduit dans les discours des fact-checkeurs en technique d'«impartialité ») et sur des pratiques de communication laissant place à l'intersubjectivité, par la proximité et l'échange avec le public, et par la prise en compte de ses attentes. Ces rubriques s'inscrivent notamment dans les stratégies de re-légitimation d'une sphère journalistique bousculée, dans sa compétition pour l'audience, par la multiplication d'énoncés concurrents au sein d'une économie de l'attention (Boullier, 2009) désormais dominée par de nouveaux acteurs de la distribution des messages : les plateformes sociales.

8 Le propos de cet article est donc d'analyser le rapport des services de fact-checking aux «théories du complot » et à la façon par laquelle les stratégies de contournement de cette catégorie repoussoir contribuent à réaffirmer la légitimité du travail de fabrication des "faits journalistiques». Après avoir analysé le discours des factcheckeurs à propos du "complotisme », nous examinerons comment cette catégorie opère dans la mise en œuvre des critères de sélection et de traitement des sujets à vérifier. Nous montrerons ensuite comment l'un des enjeux de ce nouveau produit 
journalistique est de rétablir un lien de confiance auprès de publics vulnérables aux "lectures complotistes de l'actualité ", d'une part en s'investissant d'une mission pédagogique et d'autre part en déployant des efforts de communication directe avec les lecteurs.

\section{Neutralité et impartialité : la posture des fact- checkeurs face aux contenus complotistes}

\section{Le « complotisme », une catégorie maniée avec prudence}

Interrogés sur la définition qu'ils donneraient des catégories « complotisme », « théorie du complot » et " contenus à caractère complotiste ", les journalistes ne semblent pas avoir développé de définition commune qui servirait de référence dans leur champ professionnel. Les éléments mis en avant dans leurs définitions sont très variables, et vont de la méfiance envers la parole autorisée (politique, médiatique, scientifique) à la croyance en l'existence d'un groupe caché qui tire les ficelles, en passant par le fait de ne pas admettre le hasard et de rechercher des explications simplistes. La prudence, en revanche, est une constante de leurs réponses : la plupart ont conscience du fait que le terme même de "complotisme » est déjà une «étiquette " ${ }^{6}$, " un jugement de valeur " qui «a une connotation négative $»^{8}$ et " peut être complètement contre-productif $»^{9}$, car c'est exprimer une forme de "mépris $»^{10}$ qui n'a pas sa place dans leur façon d'aborder les sujets et leurs interlocuteurs :

Je pense que la très mauvaise direction qui a été prise par le fact-checking c'est la caricature qui a été faite des gens à qui on est censés s'adresser en fait: la fake news, c'est soit les méchants Russes, soit les Gilets Jaunes, soit les débiles, soit les complotistes. [...] Je pense que, par définition, si on insulte les gens, on va assez difficilement échanger avec eux ou mettre en place un concours d'arguments pour avoir un débat rationnel ${ }^{11}$.

Pour certains, le terme présente aussi l'inconvénient d'être trop générique ${ }^{12}$, de mettre " tout le monde dans le même sac " ${ }^{13}$, alors que le fact-checking se veut justement un exercice de rigueur et de précision. Sans en faire une analyse scientifique, ils perçoivent bien le caractère problématique, polysémique et polémique de cette notion (Le Caroff \& Foulot, 2019). Ils affirment donc la manier avec précaution, s'interdire la plupart du temps de l'écrire dans leurs articles, et considèrent qu'elle ne servirait pas non plus à opérer de catégorisation, ou très peu.

11 Malgré ces précautions oratoires, les vocables " complotisme ", « théorie du complot » et « conspirationniste » apparaissent de diverses façons dans des « fact-checks » (articles dédiés à l'authentification d'une information). Factuel est le plus parcimonieux dans ses usages, seuls six articles utilisent ces expressions (pour qualifier respectivement une personne, un groupe, un mème, un ton, un type de fausse information) ${ }^{14}$. À la rédaction de 20 Minutes en revanche, il s'agit bien d'une catégorisation explicite, avec les motsclefs "complot» et «théorie du complot» attachés à certains articles. Depuis la création de Fake Off en mai 2017, le mot clé «théorie du complot " a été assigné à quarante-cinq articles du site, même s'il faut souligner que seulement sept de ces articles font officiellement partie de la rubrique de fact-checking, ce qui renforce l'idée d'un usage très parcimonieux de cette catégorie par les fact-checkeurs. La plupart du temps, lorsque ces expressions ne sont pas une simple reprise des termes de la question posée par les internautes ou une citation des personnes interrogées, cela semble être 
une utilisation par convention usuelle dans le discours médiatique, concernant les contre-discours sur le 11 Septembre, l'alunissage ou encore l'affaire du Pizzagate ${ }^{15}$.

Chez CheckNews, la situation est plus complexe: on observe que les adjectifs " complotiste » et " conspirationniste » apparaissent assez régulièrement et servent alors à qualifier un contenu ou une théorie, mais aussi fréquemment un site, une personne ou un groupe à l'origine de, ou propageant l'information analysée dans l'article ${ }^{16}$. Une pratique qui entre apparemment en conflit avec la prudence et les réticences exprimées. Toutefois, elle s'inscrit logiquement dans la pratique du factchecking qui consiste à remonter à la source, retracer l'historique de diffusion d'une information et cartographier ses relais. On note ainsi que les adjectifs ne sont pas utilisés pour décrédibiliser directement la source, mais plutôt pour situer la genèse de l'intox et analyser ses réseaux de diffusion, comme ici :

Itinéraire de l'intox. Cette publication est en fait la traduction mot pour mot d'une publication en anglais. Elle apparaît par exemple le 5 décembre 2013, sur Reddit. Le visuel est ensuite repris par de nombreux sites complotistes comme WorldTruth.tv. La publication est aussi traduite en espagnol, sur le site - également complotiste El Gran Engaño, le 27 mars 2014 $4^{17}$.

13 La volonté de remettre en contexte apparaît comme une justification sous-jacente de l'utilisation de ces termes. Il n'en reste pas moins qu'elle rejaillit sur l'image du site, des personnes ou du groupe en question et fonctionne bien comme une forme de labellisation infamante. L'adjectif "complotiste» est utilisé ici comme une mise en garde explicite sur la fiabilité des sites évoqués et sur leur nécessaire mise à l'écart de la légitimité proprement journalistique ou scientifique.

Par rapport au nombre total d'articles publiés et en excluant les citations, les fact-checks présentant des occurrences des termes "complotisme et complotiste", "théorie du complot » et "conspirationniste» représentent une infime minorité ${ }^{18}$, ce qui fonde le sentiment partagé par tous les fact-checkeurs de très peu traiter ces sujets.

\section{Complotiste n'est pas faux : évaluation ponctuelle contre labellisation générale}

Bien évidemment, les fact-checkeurs sont de facto dans une démarche normative, puisqu'ils établissent ce qui doit être considéré comme un fait avéré (Graves, 2016), mais ils adoptent dans leur discours une posture qui se veut ouverte, compréhensive, expliquant se mettre à la place des gens qui les sollicitent ${ }^{19}$. Plus qu'à des «théories du complot ", ils estiment qu'ils ont souvent plutôt affaire à des interrogations légitimes, des inquiétudes qui s'expriment, qui ont le droit de s'exprimer et qu'il faut prendre en compte, notamment sur la santé dans le cas des vaccins ou de l'incendie de Rouen, avec pour corollaire un " grand désir de vérité »"

Et partir du principe qu'on nous cache des vérités, ce n'est pas forcément mauvais en soi, ça s'applique aussi au journalisme de partir du principe qu'on nous raconte pas tout ${ }^{21}$.

Si un contenu présente un caractère complotiste, ce n'est donc "pas une raison pour juger de [sa] véracité », car " on peut être complotiste et avoir raison sur un point ${ }^{22}$ et toutes les conclusions ne sont pas nécessairement fausses ${ }^{23}$. De même, le fait que le contenu étudié soit relayé par une source qui diffuse fréquemment des informations fausses ou qui est connue pour ses orientations complotistes ne doit pas entrer en ligne de compte. L'exercice n'est pas simple, certains le reconnaissent, mais il est indispensable 
«de faire un peu abstraction $»^{24}$, de rester «sans préjugés ${ }^{25}$ et de ne " pas porter de jugement » sur la source ${ }^{26}$.

Même si c'est [...] un compte qu'on pourrait qualifier entre nous de complotiste [...], on doit pas juger ce compte, ou les publications de ce compte [...], on doit s'intéresser au fond de son propos, de cette nouvelle argumentation qu'on va essayer de vérifier ${ }^{27}$.

Qu'il s'agisse de «Big Pharma » ou des " antivax » il faut interroger toutes les sources possibles, avec une même prudence ${ }^{28}$, car dans le processus de validation, seule la véracité du contenu doit être éprouvée par une procédure méthodique. On peut avoir un sentiment de décalage entre le discours et la pratique sur ce point dans le cas de CheckNews (supra), même s'il faut souligner que certains articles confirment bien la véracité de messages relayés par des comptes qualifiés par ailleurs de "complotistes " 29 . La catégorisation de la source ne préjuge donc pas de l'issue du traitement, conformément aux engagements des cellules. Ce principe de dissociation, dans les messages analysés, entre ce qui relève de l'information factuelle, de sa source et de son cadre interprétatif, est l'un des moyens de mise en œuvre de l'impartialité revendiquée.

\section{Innover pour réaffirmer les valeurs traditionnelles du journalisme}

Le fact-checking s'affirme comme un genre journalistique qui met en place sa propre déontologie et possède son instance de certification, l'IFCN. C'est sur cette instance que Facebook s'appuie pour sélectionner des partenaires jugés fiables. Pour être membre de l'IFCN, il faut obtenir un agrément qui repose sur le rapport d'une personnalité extérieure, experte du fact-checking - en France, c'est surtout le journaliste et universitaire Laurent Bigot qui s'en charge -, qui évalue un ensemble de critères correspondant à l'application des cinq piliers de la charte élaborée fin $2016^{30}$ : impartialité et équité (1), transparence des sources (2), des financements et de l'organisation interne (3), de la méthodologie (4), respect de la politique de correction (5). Ces critères mettent en exergue des éléments de la déontologie journalistique déjà formalisés dans la Charte de Munich et la Charte d'éthique professionnelle des journalistes pour les points un et cinq, et dans différentes chartes nationales, textes internes aux médias et guides des bonnes pratiques pour les points deux à quatre (Laville, 2017).

Toutes les personnes interrogées réaffirment - par le discours, comme par les pratiques -, ces normes déontologiques incorporées à un ethos professionnel de factcheckeur qui a la particularité de devoir être exemplaire, explicite et didactique. Elles mettent ainsi systématiquement en avant l'absence de parti pris : il ne «faut pas avoir de biais ${ }^{{ }^{31}}$, équilibrer le traitement des différents camps politiques, essayer d'être "vraiment le plus neutre possible», y compris "dans la façon de rédiger » ${ }^{32}$, et "rester extrêmement factuels » dans son analyse ${ }^{33}$, ce qui explique la prudence vis-à-vis de termes connotés comme "complotisme ". Cette revendication très forte sert aussi à valoriser un groupe social qui tend à se constituer comme une spécialité à l'intérieur de la profession, et participe d'une construction symbolique d'autant plus nécessaire que le journalisme fait face à une représentation sociale de plus en plus dépréciative et que la position des fact-checkeurs dans la hiérarchie de la profession n'est pas assurée (Bigot, 2017b). 
Une fois ces principes posés, encore faut-il déterminer ce qui est traitable dans le cadre du périmètre de compétences du fact-checking.

\section{La sélection des sujets : le double filtre du « factuel » et du « viral»}

21 Les journalistes enquêtés considèrent qu'il n'y a dans leur rédaction aucune ligne éditoriale ou consigne énoncée en matière de complotisme, que ce soit pour le traiter en priorité ou au contraire pour l'écarter. Aucun contenu n'est exclu a priori et tout est traité «au cas par cas $»^{34}$. Mais de fait, ils estiment que dans leur pratique ils traitent finalement assez peu les sujets qu'ils considèrent comme étant à caractère complotiste, car cela dépasse leur champ de compétences ${ }^{35}$. En effet, peu de ces contenus passent l'épreuve des deux principaux critères d'inclusion/exclusion qui opèrent dans la sélection éditoriale des sujets "fact-checkables", à savoir : 1) y a-t-il des éléments factuels vérifiables ? ; 2) le contenu a-t-il une forte visibilité ? Ces critères peuvent aussi être complétés par d'autres, comme "l'intérêt » du sujet ou son « actualité ».

\section{Le « factuel » à l'épreuve du faisable}

Les fact-checkeurs ne s'étendent pas vraiment sur les ambivalences et le caractère délicat de la distinction entre faits et opinions, ou encore sur l'instabilité des données scientifiques et institutionnelles sur lesquels ils se fondent pour prononcer leur verdict, qu'ils doivent énoncer dans le langage du vrai et du faux. Ils tracent des lignes opérationnelles qui leur servent de boussole dans leur pratique et doivent leur éviter deux écueils: les impasses (impossibilité de se prononcer) et les sorties de pistes (entrer sur le terrain de l'opinion et de l'interprétation). Les catégories avancées se veulent claires: pour eux, le fact-checkable "c'est tout ce qui est vérifiable", "factuellement étayable ou infirmable" - même si cette définition "est un peu tautologique $»^{36}$. Est par conséquent exclu ce qui relève du jugement et de l'opinion, de l'avenir, « $d u$ procès d'intention $»^{37}$ et du secret ${ }^{38}$. Or ce dernier est l'un des principaux ressorts du complotisme. "Contrer factuellement les théories du complot, c'est compliqué " ${ }^{39}$, et ce pour plusieurs raisons : les questions sont souvent «trop larges »" il n'y a "rien de tangible $»^{41}$ sur quoi appuyer la vérification, et souvent ce ne sont que des accusations "de servir tel ou tel intérêt »". Le fonctionnement même du fact-checking impose d'apporter la preuve de ce que l'on avance pour infirmer ou confirmer la véracité d'une information, or il est impossible de démontrer l'inexistence de quelque chose, a fortiori lorsque cette chose est par définition dissimulée, comme un complot: "c'est undebunkable ${ }^{43}$, parce qu'une société secrète, par essence, on peut pas dire 'non elle existe pas'»(AFP1) ${ }^{44}$.

À l'AFP, un petit groupe de fact-checkeurs a réfléchi à la façon dont ils pourraient par exemple aborder le thème des chemtrails ${ }^{45}$, pour en conclure à l'impossibilité de traiter ce sujet dans le cadre de leur activité :

On est un peu coincés, parce que : qui on va interroger ? Est-ce qu'on va interroger un vaste panel de pilotes d'avion, des gens qui travailleraient dans l'industrie aéronautique, des scientifiques qui travailleraient sur la qualité de l'air, enfin plein d'experts, et même si on avait tous ces gens-là, à partir du moment où des gens qui seraient complotistes pourraient penser que tous ces gens-là ne sont pas de bonne 
foi et nous cachent quelque chose, comment on arrive à les convaincre avec une démonstration, j'ai envie de dire mathématique, comment on peut leur prouver que tout cela est faux ? ${ }^{46}$ interrogées, on lui rétorquera qu'elles ne sont «pas de bonne foi » ou manipulées, et qu'il fallait en chercher d'autres ${ }^{47}$. Les interviewés en arrivent parfois à la question de la confiance accordée aux scientifiques, aux institutions et aux données dont ils peuvent disposer ${ }^{48}$, mais ils ne semblent pas enclins à approfondir le sujet. Le fact-checking est une forme de production de connaissance (Graves, $2016: 69$ ) qui s'appuie explicitement et ostensiblement sur des sources institutionnelles pour formuler un jugement de véracité sur une assertion. Les verdicts énoncés reposent sur des données institutionnelles, sur le consensus scientifique - quand il existe -, et la cohérence des indices rassemblés (Graves, 2017). Par la sélection qu'il opère dans les sources, en retenant celles qu'il juge fiables, en choisissant des standards plutôt que d'autres, le fact-checking participe à l'élaboration collective de la réalité. S'ils sont conscients de leur dépendance aux sources institutionnelles et au mode de production des données utilisées (Vauchez, 2019), les fact-checkeurs l'acceptent cependant sans la remettre en question, « évacuant toute perspective constructiviste » et créant de facto une forme de conservatisme (Joux et Gil, 2020).

La frontière théorique du fact-checking est donc celle qui sépare les faits de leur interprétation (théorie, opinion, jugement), même si cette frontière est en pratique poreuse et impossible à tracer (Graves, $2016 ; 132-142$ ). Tant qu'il s'agit pour les factcheckeurs de traiter des éléments considérés comme factuels, ils peuvent vérifier, expliquer, remettre en contexte si besoin. Cela relève de leur domaine de compétence :

On a eu des choses complotistes ou à relents complotistes, qu'on a pu traiter. Je prends le cas de Rothschild, des articles qui disaient [...] «voilà, la Russie, croissance forte en Russie depuis que Poutine a fait fermer les banques Rothschild ». Et donc, là factuellement, on s'est dit " y'a quelque chose à vérifier » ${ }^{49}$.

De petites briques constitutives peuvent ainsi être abordées, de façon spécifique, isolée, comme les rumeurs qui ont circulé au moment de l'incendie de Notre-Dame de Paris ${ }^{50}$, ou même dans le cas du 11 septembre : avec les « $x$ et x points d'entrée pour dire "les tours se sont effondrées comme ça, c'est pas possible", "on savait que tel terroriste, etc.", là on peut prendre des points un par un et dire "ça c'est faux", donc c'est comme ça qu'on va attaquer les complotistes ${ }^{51}$. En revanche, lorsque le passage à la théorie se fait, c'est-à-dire lorsqu'il y a une construction idéologique des rumeurs, exactes ou non, orientées pour produire un récit politique (Campion-Vincent \& Renard, 2015; Taïeb, 2010), la frontière est franchie et le fact-checking se déclare incompétent. Il valide des faits en les confrontant à d'autres faits établis et validés par une parole " autorisée ». En dépassant ce cadre, la «théorie du complot» bascule, pour les fact-checkeurs, dans un autre registre d'énonciation, celui des représentations sociales, des mythes, croyances et idéologies.

\section{Le critère de « viralité » : intérêt public ou intérêt du public ?}

27 L'audience captée par une information sur le web est le second critère avancé par les fact-checkeurs dans le processus de sélection des sujets. Cette information fournie par les plateformes, et notamment par l'interface élaborée par Facebook, est l'une des premières choses que les fact-checkeurs examinent, même si aucun enquêté n'a su définir avec précision le seuil de pertinence. Cela relève du sens pratique des 
journalistes mis en œuvre dans la sélection des nouvelles (Charron \& De Bonville, 1997 : 57). Pour une parole publique, comme celle d'un ministre, l'importance est acquise ${ }^{52}$. Pour d'autres énoncés, cela dépend du nombre d'interactions objectivées sur les médias sociaux (vues, likes, partages), mais aussi de publications similaires et de supports différents. Cela dépend également du nombre de questions reçues directement à la cellule par des canaux de plus en plus diversifiés (site web, comptes Facebook et Twitter, email...). L'évaluation se fait au cas par cas, selon ces critères combinés, qui ne doivent pas nécessairement être tous remplis. Il n'y a donc pas de règle fixe, mais plutôt des effets de "seuil psychologique » ${ }^{53}$ : pour résumer les chiffres avancés par les journalistes interrogés, à partir de trois questions reçues directement cela devient significatif, à partir de mille partages d'une publication sur une plateforme sociale on peut se pencher sur la pertinence de sa vérification. À partir de 10000 il devient presque évident pour les fact-checkeurs qu'il faut s'emparer du sujet ${ }^{54}$.

Si cette estimation de l'audience est primordiale, c'est qu'en arrière-plan, il y a toujours la crainte de desservir la cause que l'on défend:

Ce qu'on peut reprocher aussi au fact-checking c'est que, de fait, ça met en lumière, ça fait exister la vision complotiste de tout événement. [...] [II] rend visibles des choses fausses que les gens n'avaient pas forcément vues avant, et du coup y'a une question de savoir si le remède est pire que le $\mathrm{mal}^{55}$.

Il s'agit donc toujours de mettre en balance la visibilité déjà obtenue et celle que le factchecking induira mécaniquement, "parce que rien que dans le titre on va rendre public" quelque chose qui était confidentiel ${ }^{56}$. C'est pourquoi les fact-checkeurs regardent aussi la "viralité », la dynamique de circulation des messages. Un indicateur introduit en 2019 dans l'outil Facebook permet de voir le nombre de partages dans les dernières 24 heures, ce qu'ils jugent très utile pour relativiser l'audience de certaines publications, qui peuvent comptabiliser des nombres de partages très élevés, mais anciens. La traiter reviendrait alors à lui donner une visibilité qu'elle n'a peut-être plus. Inversement, un contenu relativement peu vu, mais dont la vitesse de propagation est élevée, témoigne d'un fort "potentiel de viralité ${ }^{57}$ qui peut justifier un traitement. Les fact-checkeurs développent aussi parfois des techniques spécifiques visant à minimiser les risques de visibilisation : ils donnent la source mais «évitent au maximum de renvoyer vers le site web directement $~^{58}$, ou changent leur format, de façon à lever toute ambiguïté :

On évite de titrer sous forme de question, on va changer notre règle et titrer par « Non virgule », [...] pour éviter que le gens voient juste le titre sans cliquer et du coup se disent « ah ben oui, c'est vrai vu qu'y a une question », non ${ }^{59}$.

Si la question est posée par mail et que le contenu est très peu visible sur internet, « il vaut mieux répondre individuellement en expliquant, plutôt qu'en faire un [papier] $\aleph^{60}$. Le factcheckeur se donne ainsi pour mission morale de casser ou limiter les dynamiques de diffusion des fausses informations et assume ainsi explicitement de jouer un rôle dans la «dynamique de publicisation et de confinement de la controverse » (Lemieux, 2007 : 200).

\section{«Vieilles théories » enracinées vs. « lecture complotiste » de l'actualité}

31 Les fact-checkeurs interrogés opèrent souvent une distinction entre les "vieilles " théories du complot et les nouvelles lectures complotistes. Il y a "différents niveaux " ${ }^{61}$, une sorte d'échelle subjective, dominée par "des trucs très très complotistes $\|^{62}$, «ces 
espèces de vieilles théories du complot qui traînaient depuis des lustres sur internet » ${ }^{63}$, "des choses qui sont là toujours, qu'on retrouve tout le temps $»^{64}$, qui finalement relèvent de la "croyance $"^{65}$, tels que 11 Septembre, chemtrails, Illuminati, platisme, alunissage, Bilderberg, etc. Ces théories sont relativement circonscrites et répondent bien à la caractérisation de "connaissances stigmatisées " car, ne passant pas l'épreuve des instances de légitimation, elles restent "dans les profondeurs $d u w e b »^{66}$, dans des cercles relativement restreints, et ne "circulent pas dans le même contexte, pas forcément dans les mêmes bulles » que les nouvelles théories en lien avec des événements récents ${ }^{67}$. Elles ne parviennent pas à occuper le premier plan, même sur les médias sociaux, et n'atteignent ainsi ni la visibilité, ni le caractère d'actualité qui en feraient des sujets légitimes pour le fact-checking.

32 En revanche, la plupart des fact-checkeurs observent qu'aujourd'hui, tous les événements d'actualité peuvent donner lieu à des interprétations qu'ils qualifient de "narratif complotiste $»^{68}$, de "façon de penser complotiste " ${ }^{69}$, ou encore de "lecture complotiste $»^{70}$. Elles se nourrissent selon eux du cocktail explosif de l'inquiétude légitime face à l'événement, couplée à une méfiance à l'égard des discours officiels et médiatiques ${ }^{71}$, qui s'exprime sous la forme " on nous ment, on nous dit pas la vérité $»^{72}$, « on nous cache des choses ", et que l'on "voit ressurgir dès qu'y a un événement d'actualité un peu marquant $»^{73}$ :

Incendie de Notre-Dame, sidération, le lendemain première théorie du complot. C'est systématique, aujourd'hui il n'y a pas d'information qui échappe au bout d'un moment à des théories ${ }^{74}$.

33 Le complotisme est ainsi également perçu comme une grille d'interprétation qui va nécessairement faire son apparition, parce que « la théorie du complot apporte des réponses finalement simples qui éclairent d'un coup ${ }^{75}$. Les fact-checkeurs estiment pouvoir traiter ces interprétations en lien direct avec une actualité, parce qu'ils les voient apparaître, reçoivent immédiatement de nombreuses questions, et parce qu'elles se déclinent en éléments épars qu'ils peuvent démonter un à un ${ }^{76}$, voire anticiper :

On sait maintenant, quand y'a un truc type Notre-Dame, incendie de Rouen, on surveille de suite, on sait qu'il va y avoir des fausses informations, on sait qu'il va y avoir peut-être une théorie du complot - parfois on les devine même à l'avance hein, rapidement nous on peut l'inventer la théorie, dire «ben regardez, Chirac il était déjà mort depuis une semaine, mais bon, ils attendaient le bon moment ${ }^{77}$.

Par ailleurs, ces «lectures complotistes» de points spécifiques de l'actualité ne circulent pas de la même façon que les "vieilles théories du complot »: ces dernières ont leur nébuleuse de sites, des "sphères" identifiées ${ }^{78}$ (LIB5) et circonscrites, alors que les premières circulent de façon massive sur les médias sociaux, portées par l'actualité du sujet. Elles émanent plutôt de particuliers isolés et remontent aux factcheckeurs par des questions ou des signalements d'utilisateurs confrontés à ces contenus :

Sur Twitter ils ont vu ça [l'incendie de Notre-Dame], sur Facebook ils ont vu ça et donc ils nous interrogent là-dessus ${ }^{79}$.

Le troisième journaliste interrogé à l'AFP donne l'exemple de la rumeur sur les voitures brûlées à Paris qui seraient le résultat d'un complot des autorités pour décrédibiliser le mouvement des Gilets Jaunes : ce ne sont ni des sites identifiés comme " complotistes » qui l'ont propagée, ni des médias, mais «a priori des comptes juste de gens plus ou moins militants ». Le post a ensuite fait l'objet de reprises par d'autres personnes, peutêtre pour créer elles-mêmes un post viral et faire du buzz, et la rumeur a enflé. 


\section{Comment se constitue un fait journalistique ? "Transparence » et " pédagogie » contre défiance et inquiétudes}

Une fois que certains contenus signalés ont passé le filtre de la sélection, la question se pose de savoir concrètement comment les aborder. Les fact-checkeurs ont le rôle délicat de « décider de ce qui est vrai » et s'affirment progressivement dans le tissu des institutions qui participent à la construction sociale de la réalité publique (Graves, 2016). Ils ne l'expriment certes pas de façon aussi directe, mais tous soulignent l'exigence de rigueur et de neutralité de cette activité, leur ambition d'être une référence, qui fournit des informations aussi fiables que possible, dans une société où la défiance envers les médias alimente la migration des publics vers d'autres sources d'information. Or, il faut noter que la confiance du public est le seul fondement qui puisse justifier leur position de "juge du vrai », car elle est à la fois condition de légitimité et résultat escompté de l'exercice de leur activité. Pour les fact-checkeurs, cette confiance repose sur l'explicitation de la rigueur méthodologique de leur procédure de vérification et du travail qu'elle représente. Ce faisant, il s'agit aussi de faire reconnaître le savoir-faire d'un métier présupposant à la fois des compétences acquises et du temps dédié à leur mise en œuvre.

\section{Le temps de la vérification comme gage de sérieux}

Un papier nécessite donc du travail et surtout beaucoup de temps (parfois plusieurs jours), temps qui peut parfois être perdu si jamais le journaliste ne parvient pas à rassembler des éléments qu'il juge suffisamment fiables pour se prononcer. Le factchecking est donc soumis à une temporalité différente de celle de l'édition papier quotidienne ou de l'édition web, ce qui est parfois perçu comme un "luxe ${ }^{80}$ ou du moins une position à part ${ }^{81}$, relativement épargnée par l'injonction à la réactivité.

Le fact-checking prend ainsi consciencieusement ses distances avec les dynamiques commerciales du flux continu, de l'accélération de la production de l'information et de sa reprise quasi-instantanée (Cagé, 2015), qui risquent de faire " perdre à l'information originale toute valeur commerciale»(Cagé et al., 2017: 55). À rebours de cette tendance, il est justement utilisé pour redonner de la valeur à l'information, désormais certifiée "vérifiée » par des professionnels selon une procédure dont la rigueur est exposée. L'AFP fait ainsi de la production de son blog Factuel un argument lors de la renégociation de ses contrats auprès de ses clients ${ }^{82}$ et Libération met en avant le service CheckNews dans ses offres d'abonnement (illustration 1), tout en utilisant la newsletter gratuite du service comme une vitrine pour générer des abonnements (illustration 2). 
Illustration 1 : extrait d'un e-mail promotionnel de Libération envoyé le 23/09/20 aux abonnés à la Newsletter de CheckNews. (" 2 mois d'abonnement offerts avec nos offres spéciales ! »)
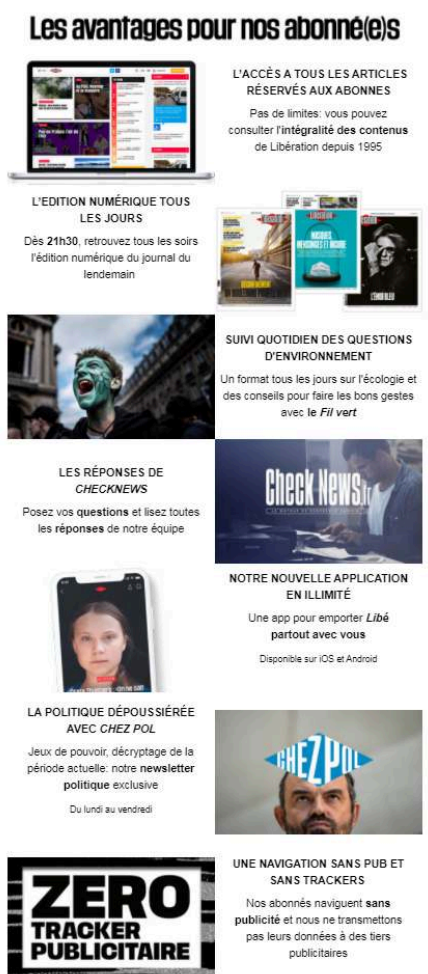

Illustration 2 : en-tête et pied de page de la newsletter CheckNews du 25/09/20.

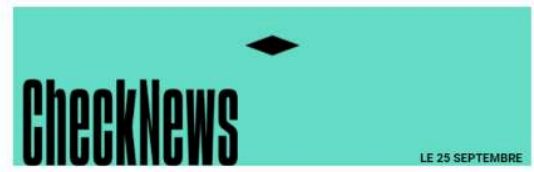

Soutenez CheckNews

Pour soutenir le travail quotidien de fact-checking du service

CheckNews, accéder en iimitie à loutes les rêponses apportées à vos

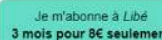

Ios Qullestions. nos réponolsegs

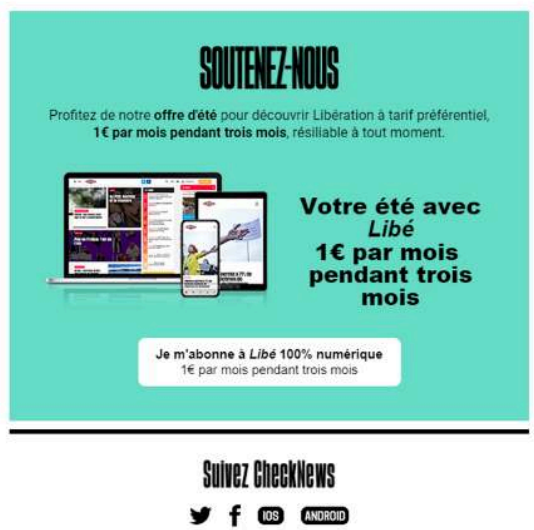

39 L'enjeu stratégique de cette activité est donc de reconstituer le capital symbolique des médias d'actualité. Et les rédactions cherchent bien à tirer parti de la crédibilité et du 
succès du fact-checking, dotant leurs cellules d'un nom distinct et souvent de leur propre logo, qui en font des marques à part entière (Bigot, 2018). L'objectif est d'atteindre une certaine notoriété auprès du public : «nous ce qu'on aimerait faire, c'est que les gens se disent : bon ben si CheckNews dit ça, c'est qu'ils ont pris le temps de vérifier $»^{83}$.

\section{Expliquer, donner des clés et dévoiler}

Les fact-checkeurs interrogés s'attribuent une fonction pédagogique, dans une relation qu'ils voudraient non pas verticale mais collaborative. Leur démarche vise à rendre les lecteurs autonomes et compétents dans la recherche d'informations :

On leur donne les outils pour faire eux-mêmes cette démarche s'ils le souhaitent. C'est ce qu'on essaye de faire en expliquant avec quel outil on travaille, comment on travaille, parce que les outils avec lesquels on travaille sont quasiment tous open source et disponibles pour n'importe quelle personne sur le web ${ }^{84}$.

41 Cette pratique correspond au deuxième point de la charte de l'IFCN, qui précise que les lecteurs doivent avoir suffisamment de sources pour pouvoir "vérifier par euxmêmes $\|^{85}$. Les fact-checkeurs reprennent ainsi à leur compte la promotion de l'éducation aux médias, préconisée depuis 1982 par l'UNESCO ou plus récemment par le ministère de l'Éducation Nationale, aussi bien pour lutter contre le complotisme que pour rétablir la confiance envers les médias et la démocratie ${ }^{86}$. Expliquer comment l'information est produite et vérifiée, éduquer à l'image et à l'esprit critique pour que le public interroge la forme et les sources de ses informations, sont autant d'arguments militant pour la légitimité du journalisme professionnel - légitimité qu'il emprunte ici au domaine de l'éducation, cette fonction pédagogique lui conférant une certaine noblesse.

C'est aussi cette approche qui permet de justifier le traitement de certains sujets et sert de cache-sexe à des impératifs moins nobles (audience et divertissement). On sent en effet parfois chez les fact-checkeurs un malaise né de la tension entre la futilité de certains sujets viraux, que la pression des chiffres de diffusion les oblige à traiter, et leurs idéaux professionnels: comment concilier le fact-checking d'un banal cliché d'animaux truqué, sujet jugé en soi peu intéressant ${ }^{87}$ mais extrêmement viral, et les exigences d'une qualité éditoriale que personne ne veut brader? Mettre en scène la relation pédagogique est une façon avantageuse de résoudre cette tension. Ils peuvent ainsi « faire des choses un peu plus légères si c'est très viral et que derrière on peut expliquer une méthodologie, on peut expliquer comment on arrive à voir que c'est photoshopé, voilà, donc ça nous est arrivé de faire des histoires de lions $»^{88}$.

43 Les questions qui parviennent à CheckNews révèlent aussi une demande de transparence accrue de la part du public, qui interroge souvent sur le fonctionnement de la rédaction de Libération, sur le travail de la cellule et sur ses relations aux nouvelles superpuissances du capitalisme informationnel :

On a aussi la volonté d'expliquer vraiment en quoi consiste le partenariat avec Facebook parce que c'est pas que du complotisme, c'est juste de la défiance qui peut être normale aussi de se dire "ils travaillent avec les GAFA c'est bizarre », mais de vraiment expliquer à chaque fois notre partenariat pour pas être accusés de servir un intérêt ${ }^{89}$.

Les fact-checkeurs considèrent cette transparence comme un préalable indispensable au rétablissement de la confiance vis-à-vis des médias ${ }^{90}$. Leurs articles sont le lieu de 
développement d'un métadiscours journalistique qui, en dévoilant au public les dessous du métier, participe explicitement d'une stratégie de reconquête de l'audience :

On détaille toujours toutes nos méthodes, y'a pas de secret, comment on a fait, c'est pas «oui c'est faux parce que c'est comme ça » [...]. Notre métier est tellement en crise que ça suffit pas d'être bon: faut être bon, faut être transparent, faut se demander «je peux faire le meilleur reportage du monde, est-ce qu'il va être lu? qui est-ce qu'il va toucher ? est-ce que je m'y prends de la bonne façon ? ${ }^{91}$

Réinvestissant le mythe fondateur de la "vertu pédagogique ${ }^{92} \mathrm{du}$ journalisme (Le Bohec, 2000), le fact-checking entend éclairer son public sur le monde en fournissant une information fiable, mais aussi éclairer sur les mécanismes de l'information elle-même. Il contribue ainsi à établir une forme de contrôle sur l'interprétation de leur activité qui lui permet d'en faire la promotion et d'entretenir sa légitimité.

Les fact-checkeurs interrogés semblent croire en l'efficacité de cette nouvelle relation pédagogique et beaucoup disent constater des changements d'usages chez les internautes, qu'ils trouvent "plus prudents, plus circonspects ${ }^{93}$ vis-à-vis des fausses informations et manifestant désormais plus souvent "des réflexes de vérification ou en tout cas de doute ${ }^{94}$. L'évolution des discussions dans les groupes de Gilets Jaunes sur Facebook leur semble aussi flagrante: il ne serait aujourd'hui plus possible de faire passer «un truc complètement complotiste délirant» sans que les gens réagissent, s'interrogent, postent des fact-checks :

Je pense qu'on peut s'enorgueillir d'un truc - tous les sympathisants Gilets Jaunes sont en général des gens qui sont en défiance extrême par rapport aux médias - on n'aura pas fait regagner la confiance, mais on aura inculqué au moins, je pense, la culture du doute, et ça c'est hyper important ${ }^{95}$.

Cette évolution témoignerait de "la vertu du fact-checking » et de la contribution au programme de Facebook. Même si ce dernier ne fournit pas de chiffres sur la diffusion et l'impact de leurs articles, les fact-checkeurs disent percevoir une tendance encourageante.

\section{La communication des fact-checkeurs : stratégies de (re)conquêtes et de légitimation}

48 Le journalisme du modèle biface (publicitaire) repose sur une triple fidélisation des producteurs de contenus, des annonceurs publicitaires et de l'audience. Face à la fuite des ressources publicitaires, aspirées par les grands acteurs du numérique, à la réduction et la précarisation des effectifs des rédactions (Cagé, 2015), à la crise de la représentation et à la perte de confiance dans les médias, le journalisme élabore des stratégies de valorisation à la fois symbolique et économique, dans lequel le factchecking trouve toute sa place.

\section{"Qui nous lit ? » Le public, cet inconnu à (re)conquérir}

Dans cette perspective, les fact-checkeurs mobilisent des représentations du monde social qui leur permettent de décrire leurs publics et d'ajuster leur manière de s'adresser à eux. Pour ces journalistes, en matière de complotisme, de même qu'il y a une échelle des théories, il y a aussi une distinction entre les publics selon un «gradient d'adhésion » (Le Caroff et Foulot, 2019), partagée par à peu près tous les 
acteurs interrogés, et que le premier journaliste interrogé chez Libération exprime très clairement :

Si on schématisait, y'a les gens qui propagent et qui créent, enfin, qui structurent la théorie du complot. Eux ils sont [...] tellement convaincus de ce qu'ils font, de leur bien-fondé, que jamais ils croiront les médias qui de toute manière font partie du grand complot qu'ils dénoncent ; après, et c'est à eux que nous on essaye de parler, y'a tout un ensemble de gens qui ont relayé, qui ont liké peut-être, qui ont fait un commentaire, mais qui ont jamais écrit un article, qui vont jamais produire euxmêmes un contenu, c'est à ces gens-là que nous on essaye de s'adresser, en tout cas c'est pour eux qu'on estime que notre papier a le plus d'intérêt, et pour tous les gens qui sont indécis aussi.

Toucher le public des contenus complotistes reste compliqué, car ce qui le caractérise est justement sa grande défiance envers les médias professionnels au sein desquels les fact-checkeurs exercent leur activité (Le Caroff et Foulot, 2017). Et c'est là selon eux que réside la principale utilité de l'outil Facebook, jugé « très appréciable, parce qu'au moins on peut adresser quelque chose à ces personnes ${ }^{96}$, "c'est le meilleur moyen qu'on a jusque-là de les toucher $"{ }^{97}$. En effet, le programme joint automatiquement les fact-checks aux publications classées fausses ou trompeuses, envoie une notification à ceux qui les ont déjà partagées et un avertissement à ceux qui veulent les partager.

Illustration 3 : exemple d'affichage sur un mur Facebook, lorsqu'une publication a été vérifiée et classée dans la catégorie « faux » par des cellules de fact-checking du programme de Facebook. Accès : https ://www.facebook.com/thierry.alaux.11/posts/2310783152487792, consulté le 24/11/19.

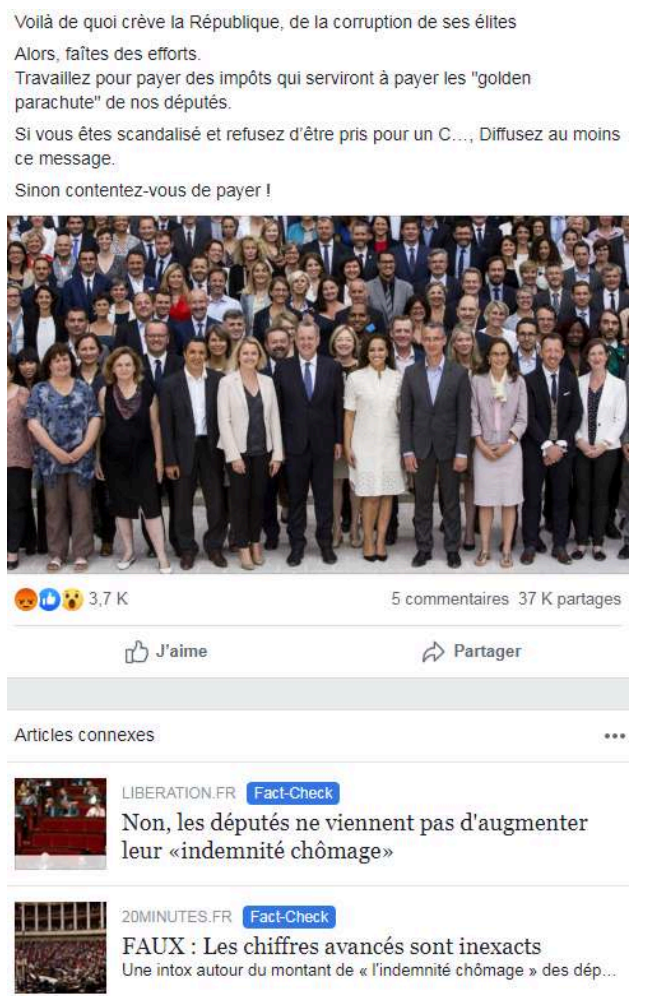


Illustration 4 : exemple de notification affichée lorsque l'utilisateur de Facebook veut partager cette publication. Accès : https ://www.facebook.com/thierry.alaux.11/posts/2310783152487792. consulté le 24/11/19.

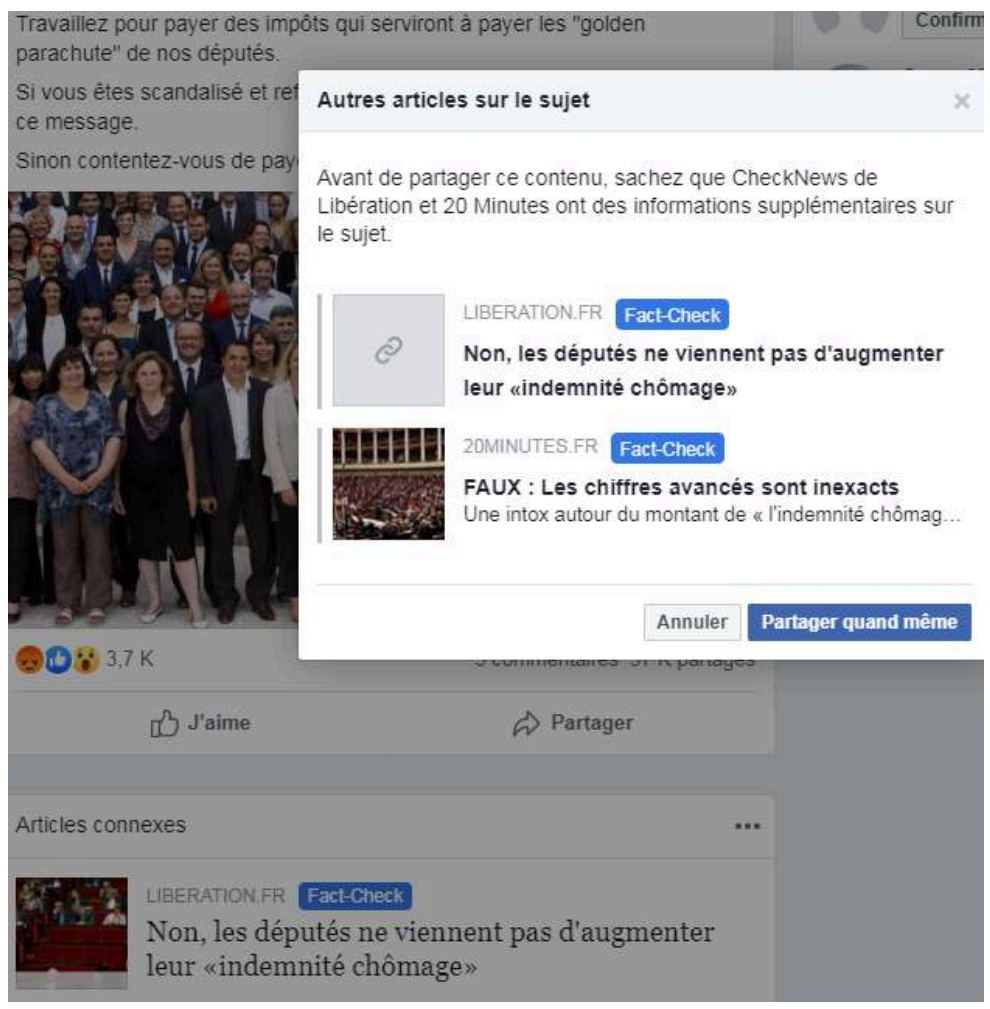

51 Ce dispositif adresse donc des messages à la fois ciblés et systématiques. Certains factcheckeurs travaillent aussi de façon très artisanale, en mettant manuellement un lien vers leur article dans les commentaires sous une publication fausse très partagée ${ }^{98}$. S'insérer ainsi directement dans le flux des plateformes et gagner en visibilité sur les moteurs de recherche par les techniques de référencement semble être une des stratégies privilégiées pour tenter de s'adresser au public effectif des contenus à caractère complotiste. Il y a en tout cas chez les journalistes interrogés un sentiment de s'adresser à des audiences qui habitent des mondes différents, où chacun s'enferme dans des «bulles informationnelles» (Pariser, 2011). Arriver à faire en sorte que «ces bulles se croisent à un certain moment » est tout l'enjeu du fact-checking ${ }^{99}$, même si cela ne garantit pas l'adhésion. Les acteurs savent en effet que leur vérification, même diffusée de façon ciblée, ne pourra pas « convaincre tout le monde »100.

\section{« Proximité » et « neutralité », conditions de la confiance}

52 Le secteur du journalisme «est sinistré économiquement [...] parce que les gens lisent plus, parce qu'ils croient plus» et que "la défiance est gigantesque " ${ }^{101}$. Dans ce contexte, aucun des acteurs ne voit le fact-checking comme la panacée qui sauvera leur profession de la crise ${ }^{102}$. Il est plutôt perçu comme une forme "complémentaire »" un "genre journalistique» parmi d'autres ${ }^{104}$, «un moyen parmi plein d'autres de rétablir la confiance $»^{105}$.

53 Cela passe, pour les fact-checkeurs, par la proximité et l'interactivité, la conversation publique sur les réseaux sociaux ou privée par email. Se sentant «très proches de leurs lecteurs $»^{106}$, «en contact avec les lecteurs de 'base' $»^{107}$, « en prise directe avec les réseaux 
sociaux $»^{108}$, les fact-checkeurs apprécient cette position charnière de relations publiques. C'est l'une des spécificités qui les distingue de certains de leurs confrères. Chez CheckNews, le mode de fonctionnement adopté par la cellule depuis 2017 en a même fait une forme d' « interface entre les lecteurs et le journal » ${ }^{109}$. En passant du modèle traditionnel de Désintox, où les fact-checkeurs scrutaient uniquement la parole publique pour y dénoncer le "faux", au modèle plus interactif de CheckNews, qui répond exclusivement aux sollicitations extérieures, l'objectif était bien d'« imaginer un espace de discussion, une espèce de passerelle vers des gens avec qui on parlait pas ", ce qui redonne du sens à leur activité110. Les fact-checkeurs se veulent à l'écoute des besoins des lecteurs, tiennent à répondre à leurs attentes, sans juger leurs centres d'intérêt, sans endosser le rôle traditionnel du gatekeeper qui filtre en amont l'information et prescrit l'actualité en fonction de l'importance qu'il décide de lui accorder. Cela fait partie du respect qu'ils estiment devoir à leurs interlocuteurs et au public en général. "On s'empare de tous les thèmes, du plus léger au plus grave $»^{111}$, en affirmant que tout le monde a droit à une information vérifiée :

Faut pas que les choses sérieuses et sûres ne concernent que des sujets sérieux.

Même pour les sujets futiles les gens ont droit à avoir des articles sérieux ${ }^{112}$.

Ceci pose de fait la question des choix éditoriaux des cellules de fact-checking. Elles se heurtent, selon les enquêtés, à l'incompréhension de certains collègues, qui continuent à aborder leur activité sur le principe d'une production collective de l'actualité, d'un regard sélectif sur le monde propre au champ journalistique (Bourdieu, 1994 : 5). Et, au sein de ce pôle «noble » ou " élitiste » de la presse nationale d'opinion (comme ici à Libération), les fact-checkeurs produisent une dissonance entre la promotion d'une orthodoxie méthodologique (qui dans l'idéal est partagée avec leurs collègues), et son application occasionnelle - sous couvert de pédagogie - à des sujets illégitimes, dans une relation qui ne se veut pas surplombante, ni en rapport aux sources, ni dans la relation avec le public. Dans un organe comme Libération, qui revendique son engagement, la posture d'impartialité de CheckNews n'est pas sans créer quelques tensions : parfois accusée de "dépolitiser » le journalisme ${ }^{113}$, certains reprochent à la cellule «de faire des sujets trop putassiers $»^{114}$, renvoyant à une logique décriée de journalisme commercial orienté vers la demande, qui vise une audience plus que l'intérêt commun (Cornu, 2013). Pour une partie de la profession, l'intérêt que le public porte à ces sujets ne constitue pas une justification suffisante, et «si y'a des gens qui sont assez bêtes pour penser ça, c'est pas à [eux journalistes] de [s'en occuper] ${ }^{115}$. Cette posture surplombante de certains de leurs confrères est pour les fact-checkeurs une des sources du problème de la relation aux médias. Ce n'est pas en prenant les gens " de haut » ${ }^{116}$, "en leur disant frontalement qu'ils se trompent $»^{117}$ ou que "c'est absurde de penser ça $»^{118}$ qu'on peut les convaincre, au contraire, «c'est comme ça qu'on les perd »"19. Ils croient en la rationalité partagée et en la division du travail intellectuel d'objectivation factuelle à laquelle ils participent, mais aussi à leur mission pédagogique et à la capacité du public à mobiliser des ressources de façon autonome. Les fact-checkeurs que nous avons interrogés refusent de reproduire les discours de disqualification du public que l'on peut observer par ailleurs dans le cas du Décodex ou de la Journalism Trust Initiative (Doutreix \& Barbe, 2020). Ils entendent plutôt renouveler le magistère journalistique dans une relation plus horizontale et inclusive.

Cette position les amène à se concevoir comme un service au public, voire quasiment comme un "service public ». Un fact-checking qui privilégie "l'échange et le respect» peut selon eux « tisser de la confiance $»^{120}$, « recréer du lien ou une confiance avec les lecteurs, 
parce qu'il y a ce côté collaboratif ${ }^{121}$. Guichetiers des médias, prestataires de services gratuits, les fact-checkeurs répondent aussi à une demande qui s'exprime par les médias sociaux et les interfaces du journal. En accord avec cette conception servicielle, ils peuvent en venir à adresser un courrier individuel si besoin, lorsque la véracité ou la fausseté du contenu a déjà été établie. Il se dessine ici, tout comme dans la façon de répondre ou d'être interpellé sur les plateformes sociales, une relation personnalisée qui redonne chair à l'institution. Ils doivent incarner l'exemplarité, démontrer leur savoir-faire et leur rigueur impartiale :

Il faut faire la preuve de sa bonne foi en permanence, et de son ouverture d'esprit et

de pas être dans l'argument d'autorité.

De fait, on observe que la crédibilité des fact-checkeurs est fortement corrélée à la façon dont le public perçoit leur impartialité (Brandtzaeg \& Følstad, 2017). La plupart soulignent l'exigence incroyable et la fragilité extrême de l'édifice : les fact-checkeurs se doivent d'être exemplaires, il faut être une "référence ", "c'est super exigeant »"122, car "la confiance dans les médias c'est quelque chose qui est horriblement difficile à construire, [...] il suffit d'une tournure de phrase maladroite pour tout foutre en l'air ${ }^{123}$. Cette "grande vulnérabilité vis-à-vis de l'erreur " (Vauchez, 2019: 34) est le revers de la médaille d'une relation directe avec le public.

\section{Conclusion}

Les fact-checkeurs se représentent donc bien le caractère hétérogène des catégories de "complotisme» ou «théorie du complot» et prennent en compte dans leurs publications et leurs échanges avec leur public les effets contreproductifs de stigmatisation liés à l'utilisation de ces termes connotés. Ces représentations, couplées à un élément clé de leur identité professionnelle, la neutralité, explique que leur pratique se révèle généralement en accord avec la prudence affichée dans leur discours sur ce sujet. Par ailleurs, ils ne font pas de la « lutte contre le complotisme » leur cœur de métier, et ce pour plusieurs raisons : d'une part, ils estiment que tout un versant du complotisme, celui des "vieilles théories » enracinées dans des structures mythiques ou des groupes subversifs, relève de l'opinion et non seulement déborde de l'espace des possibles en termes de vérification et de production de "faits journalistiques", mais s'adresse à des publics jugés trop éloignés et inaccessibles au discours du fact-checking. Ils les repoussent donc au-delà des frontières de leur activité.

Cependant, ils traitent bien un certain nombre de contenus qu'ils jugent "à caractère complotiste » ou vérifient des informations susceptibles de "lectures complotistes", liées ou pas à des événements d'actualité, mais faisant partie d'un phénomène plus large et perçu comme nouveau : la prolifération des fausses informations. Représenté comme un danger pour les valeurs de la démocratie, ce phénomène donne lieu depuis quelques années à une véritable panique morale, largement entretenue par les médias traditionnels (Koukoutsaki-Monnier, 2018), et qui constitue une opportunité pour le journalisme professionnel de se mettre en scène pour réaffirmer ce qu'il considère comme son rôle démocratique et sa spécificité dans un paysage informationnel en recomposition (Doutreix et Barbe, 2020). Car le journaliste doit désormais se distinguer non seulement des politiques, des agences de communication et des chargés de relations publiques (Schudson, 2001), mais aussi de la masse des individus qui produisent et relaient de l'information via les médias sociaux. Ces deux éléments 
constituent aujourd'hui les "circonstances culturelles spécifiques» (Schudson, 2001) qui permettent d'expliquer la valorisation des normes d'impartialité et de factualité incarnées par le fact-checking.

Sa dimension didactique vise explicitement à armer le discernement critique des lecteurs : l'aptitude à évaluer les sources et comprendre la production de l'information serait nécessaire à la formation d'une opinion rationnelle (ou du moins raisonnée) des citoyens. Implicitement, elle fait la preuve par l'exemple du caractère nécessairement professionnel de la production d'informations (avec une temporalité, des compétences et une déontologie spécifiques). La mise en scène d'une rigueur méthodique dans la production de faits vérifiés et vérifiables s'inscrit ainsi à la fois dans une représentation fonctionnaliste qui confère un rôle structurel à la presse dans les démocraties représentatives, et dans des enjeux d'audience dont dépend en grande partie la possibilité économique d'un journalisme professionnel.

Aussi la stratégie de mise en place des «services» de vérification factuelle (un fairevaloir parmi d'autres produits éditoriaux) vise-t-elle à reconquérir des publics potentiellement séduits par de fausses informations, dont certaines présentent une "lecture complotiste", mais prédisposés à revenir dans le giron de l'autorité journalistique - que les acteurs désignent comme une relation de «confiance». Cette stratégie de reconquête s'accompagne non seulement d'une production d'un type nouveau, mais aussi du renouvellement des formes d'interaction avec le public. La dimension « conversationnelle » du travail journalistique a quitté le service spécialisé $d u$ «courrier des lecteurs " pour être incorporée dans les pratiques de la plupart des journalistes, et tout particulièrement des fact-checkeurs. En effet, si ceux-ci traitent des sujets prescrits par les plateformes sociales ou par les lecteurs, c'est aussi pour que les articles de vérification indexés aux contenus signalés (et à leur audience "virale ») fassent l'objet d'échanges directs avec le public, qui mettent en scène l'éthique journalistique et l'enjeu de la confiance, nœud central de la reconquête d'audience et du renouvellement de la légitimité journalistique.

61 Finalement, on pourrait en venir à considérer ce travail de fact-checking comme un nouveau mécanisme de gatekeeping qui, tout en évitant de stigmatiser frontalement des sources ou lectures complotistes, contribue à reprendre la main sur le travail de production des problèmes publics et à rétablir les frontières qui tendaient à s'estomper entre information et communication, entre opinion et faits journalistiquement vérifiables, entre amateurs et professionnels et entre producteurs et consommateurs d'informations journalistiques. En départageant méthodiquement le vrai du fake, en esquivant consciencieusement les "vieilles théories complotistes» ou en assistant didactiquement ceux qui seraient tentés par des lectures complotistes de l'information, ils n'en contribuent pas moins à des processus de disqualification qui réaffirment une vision conservatrice et tendent à consolider les paroles d'autorité (Joux et Gil, 2020) dans une fabrique sociale de la vérité marquée par l'histoire longue et conflictuelle de la confiance et des institutions qui la produisent (Shapin, 2007). 


\section{BIBLIOGRAPHIE}

BARKUN Michael (2015). « Les théories du complot comme connaissance stigmatisée », Diogène, 249-250, pp. 168-176.

BECKER Howard (1963). Outsiders: studies in the sociology of deviance, New York, Free Press.

BIGOT Laurent (2018). « Rétablir la vérité via le fact-checking : l'ambivalence des médias face aux fausses informations ", Le Temps des médias, 30, pp. 62-76.

BIGOT Laurent (2017a). « Le fact-checking ou la réinvention d'une pratique de vérification », Communication \& langages, 192, pp. 131-156.

BIGOT Laurent (2017b). L'essor du fact-checking : de l'émergence d'un genre journalistique au questionnement sur les pratiques professionnelles. Thèse de doctorat en Sciences de l'information et de la communication, soutenue le 7 décembre 2017, Université Paris II - Panthéon-Assas.

BoUGNoux Daniel (2007). « Médias et démocratie. La fonction des médias dans la démocratie », Cahiers français, 338 (3), pp. 3-7.

BOULLIER Dominique (2009). « Les industries de l'attention : fidélisation, alerte ou immersion », Réseaux, 2 (154), pp. 231-246.

BOURDIEU Pierre (1994). «L'emprise du journalisme », Actes de la recherche en sciences sociales, 1 (101), pp. 3-9.

BRANDTZAEG Petter Brae \& FøLSTAD Asbjørn (2017). « Trust and distrust in online fact-checking services ", Communications of the ACM, 9 (60), pp. 65-71.

BUTTER Michael \& KNIGHT Peter (2015). « Combler le fossé. L'avenir des recherches sur les théories du complot », Diogène, 249-250, pp. 21-39.

CAGÉ Julia (2015). Sauver les médias. Capitalisme, financement participatif et démocratie, Paris, Seuil. CAGÉ Julia, HERVÉ Nicolas \& VIAUD Marie-Luce (2017). L'Information à tout prix. Bry-sur-Marne, Ina. CAMPION-VINCENT Véronique \& RENARD Jean-Bruno (2015). « Avant-propos », Diogène, 249-250, pp. $2-8$.

CARDON Dominique (2019). Culture numérique, Paris, Les Presses de Sciences Po. CHARRON Jean \& DE BONVILLE Jean (1997). « Le paradigme du journalisme de communication : essai de définition », Communication, 17, pp. 51-97.

CORNU Daniel (2013). « Journalisme en ligne et éthique participative », Éthique publique [En ligne], 1 (15), consulté le 09.04.21. URL: https://doi.org/10.4000/ethiquepublique.1073.

DOUTREIX Marie-Noëlle \& BARBE Lionel (2020). « Légitimer et disqualifier : les Fake News saisies comme opportunité de normalisation du champ journalistique ", Études de communication [En ligne], 53 | 2019, mis en ligne le 01.01.2020, consulté le 20.04.2020. URL: http:// journals.openedition.org/edc/9242.

GIRY Julien (2017). «Étudier les théories du complot en sciences sociales : enjeux et usages », Quaderni, 3 (94), pp. 5-11.

GOFFMAN Erving (1975). Stigmate : les usages sociaux des handicaps, Paris, Minuit. 
GRAVES Lucas (2016). Deciding what's true. The rise of political fact-checking in American journalism, New York, Columbia University Press.

GRAVES Lucas (2017). « Anatomy of a fact check: objective practice and the contested epistemology of fact checking ", Communication, Culture and Critique, 3 (10), pp. 518-537.

HUYGUE François Bernard (2018). Fake news : la grande peur, Versailles, VA éditions.

Joux Alexandre \& GIL Inès (2020). « Entre transparence des sources et entre-soi : une critique du Fact-checking du débat de l'entre-deux tours de la présidentielle française de 2017 », Études de communication [En ligne], 53 | 2019, mis en ligne le 01.01.2020, consulté le 20.04.2020. URL : http:// journals.openedition.org/edc/9287.

KOUJOUTSAKI-MONNIER Angeliki (2018). « Narratives of the fake news debate in France », The IAFOR journal of art \& humanities, International Academic Forum, 5 (2), pp. 3-22.

LAVILLE Camille (2017). « Le journaliste, un professionnel de la transparence ? », Sens-Dessous, 2 (20), pp. 29-36, consulté le 15.09.20. URL : https://www.cairn-int.info/revue-sens-dessous-2017-2page-29.htm.

LE BOHEC Jacques (2000). Les mythes professionnels des journalistes, Paris, L'Harmattan.

LE CAROFF Coralie \& FOULOT Mathieu (2017). «Les théories du complot et leurs adhérents : approcher les usages en ligne des arguments conspirationnistes », étude présentée lors du congrès 2017 de l'Association française de science politique, consulté le 11.11.19. URL : https:// www.afsp.info/congres/editions-precedentes/congres-2017/sessions/sections-thematiques/ st52/.

LE CAROFF Coralie \& FOULOT Mathieu (2019). «L'adhésion au « complotisme » saisie à partir du commentaire sur Facebook », Questions de communication, $n^{\circ} 35$ [En ligne], mis en ligne le 01.10.19, consulté le 11.11.19. URL :

http://journals.openedition.org/questionsdecommunication/19405.

LEMIEUX Cyril (2007). «À quoi sert l'analyse des controverses ? ", Mil neuf cent, revue d'histoire intellectuelle, 25 (1), pp. 191-212.

MATHIEN Michel (2001). « Le journalisme de communication : critique d'un paradigme spéculatif de la représentation du journalisme professionnel », Quaderni, 45, pp. 105-135, consulté le 18.08.20. URL : http://www.persee.fr/doc/quad_0987-1381_2001_num_45_1_1500.

PARISER Eli (2011). The filter bubble: what the Internet is hiding from you, New York, Penguin Press. RUELLAN Denis (1992). « Le professionnalisme du flou », Réseaux, 10 (51), pp. 25-37, consulté le 07.07.20. URL : https://www.persee.fr/doc/reso_0751-7971_1992_num_10_51_1922.

SCHUDSON Michael (2001). « The objectivity norm in American journalism », Journalism, 2 (2), pp. 149-170.

SHAPIN Steven (2007). A social history of truth. Civility and science in seventeenth-century England, Chicago, Univ. of Chicago Press.

TAїEB Emmanuel (2010). « Logiques politiques du conspirationnisme », Sociologie et sociétés, 42 (2), pp. 265-289.

VAUCHEZ Ysé (2019). « Les mythes professionnels des fact-checkeurs. Un journalisme de données au service de la vérité », Politiques de communication, 12, pp. 21-44.

WEBER Max (1971). Économie et société, Paris, Plon. 


\section{NOTES}

1. Menée dans le cadre du programme interdisciplinaire PandHeMic actuellement en cours à l'Université de Caen.

2. Organisation hébergée par le Poynter Institute for Medias Studies, école de journalisme à but non lucratif et centre de recherches basé en Floride.

3. Six francophones adossés à des médias d'information (Fake Off de 20 Minutes, Factuel de l'AFP, Les Observateurs de France 24, Les Décodeurs du Monde, CheckNews de Libération et Vrai ou Fake? de France Info), et un anglophone spécialisé dans les sciences (Science Feedback).

4. Les Décodeurs n'ont cependant pas répondu à notre courrier. Les Observateurs et Vrai ou Fake? n'ont quant à eux pas été sollicités, relevant de l'espace télévisuel.

5. Ce programme compte en outre «Les Observateurs » (France 24) et «Les Décodeurs " (Le Monde). Facebook met à leur disposition une interface listant des contenus signalés par les utilisateurs et jugés suspects. Les fact-checkeurs choisissent le contenu qu'ils veulent vérifier. À l'issue de leur travail, ils le classent dans une des huit catégories proposées : faux, mélange [de vrai et de faux], titre trompeur, vrai, non éligible, satire, opinion, générateur de blagues. Pour les trois premières catégories, ils doivent justifier cette évaluation par un lien vers leur article. Ce dernier est alors attaché à la publication lorsqu'elle apparait sur le fil d'actualité d'un utilisateur de Facebook. Une notification est également envoyée aux personnes qui l'ont déjà partagée (infra, illustrations 3 et 4). Pour une description complète du dispositif, voir le rapport de l'organisation de fact-checking britannique Full Fact: Report on the Facebook Third Party Fact Checking programme, Jan-June 2019, p. 9-12. Accès: https://fullfact.org/media/ uploads/tpfc-q1q2-2019.pdf consulté le 12/11/19.

6. Entretien avec journaliste $n^{\circ} 5$ de Libération

7. Entretien avec journaliste $n^{\circ} 3$ de Libération

8. Entretien avec journaliste $n^{\circ} 1$ de Libération

9. Entretien avec journaliste $n^{\circ} 4$ de Libération

10. Entretien avec journaliste $n^{\circ} 1$ de l'AFP

11. Entretien avec journaliste $n^{\circ} 5$ de Libération

12. Entretien avec journaliste ${ }^{\circ} 1$ Libération

13. Entretien avec journaliste $n^{\circ} 2$ de l'AFP

14. Relevé effectué par recherche de mots clefs sur la page de Factuel le 20 novembre 2019, dont la présentation sous forme de fil chronologique infini rendait impossible un décompte du nombre total d'articles publiés (plusieurs centaines). Au 15 septembre 2020, l'architecture a été modifiée et plus aucun article disponible par recherche ne comporte ces termes (recherche qui ne permet plus de remonter au-delà de neuf mois et qui ne prend plus en compte que les mots présents dans les titres).

15. Le site de 20 Minutes a créé un dossier «théorie du complot» qui rassemble les articles du média relatifs à cette thématique, sans doute identifiée par la rédaction comme porteuse depuis la crise du coronavirus (accès: https://www.20minutes.fr/ dossier/theorie_du_complot). Au 15 septembre 2020, il compte 65 articles, et aucun nouvel article de Fake Off. On y trouve en revanche deux articles de "OMF Oh My Fake ", une émission lancée par 20 Minutes fin novembre 2019 sur Snapchat Discover, présentée 
comme un «programme qui vous rend fort contre les 'fake news'. [...] en analysant les mécanismes qui les rendent attractives ».

16. Dans vingt-trois cas relevés, ils qualifient une théorie ou un contenu, dans vingttrois autres des personnes, des groupes ou des sites.

17. Service CheckNews, "Non, ces neuf pays ne sont pas les seuls à ne pas avoir de 'banque centrale Rothschild'", CheckNews/Libération, 12/02/19. Accès: https:// www.liberation.fr/checknews/2019/02/12/non-ces-neuf-pays-ne-sont-pas-les-seuls-ane-pas-avoir-de-banque-centrale-rothschild_1708847, consulté le 19/11/19.

18. On en dénombrait quarante-six sur 4149 chez CheckNews au 19 novembre 2019, soit $1,1 \%$ des articles.

19. Entretien journaliste $n^{\circ} 1$ de 20 Minutes

20. Entretien journaliste $n^{\circ} 2$ de 20 Minutes

21. Entretien journaliste $n^{\circ} 2$ de l'AFP

22. ibid.

23. Entretien journaliste $n^{\circ} 1$ de Libération

24. Entretien journaliste $n^{\circ} 2$ de l'AFP

25. Entretien journaliste $n^{\circ} 1$ de l'AFP

26. Entretien journaliste $n^{\circ} 2$ de 20 Minutes

27. Entretien journaliste $n^{\circ} 2$ de l'AFP

28. Entretien journaliste $n^{\circ} 1$ de l'AFP

29. Cf. Donada, Emma, «Le message d'un « ancien chef militaire » sur les gilets jaunes est-il authentique?", CheckNews/Libération, 10/01/2019. Accès: https:// www.liberation.fr/checknews/2019/01/10/le-message-d-un-ancien-chef-militaire-surles-gilets-jaunes-est-il-authentique_1701862, consulté le 23/11/19; «Le gouvernement va-t-il punir les conversations jugées déviantes» CheckNews/Libération, 23/05/18 : «La suite de l'article publié sur le site complotiste Fawkes en septembre dernier est vraie. » Accès : https://www.liberation.fr/checknews/2018/05/23/le-gouvernement-va-t-ilpunir-les-conversations-jugees-deviantes-lien-signale-sur-facebook_1655241, consulté le 23/11/19.

30. Accès : https://ifcncodeofprinciples.poynter.org/ consulté le 20/11/19. À cette date, soixante-huit cellules de fact-checking étaient membres de l'IFCN, dont sept basées en France.

31. Entretien journaliste $n^{\circ} 1$ de l'AFP

32. Entretien journaliste $n^{\circ} 2$ de 20 Minutes

33. Entretien journaliste $n^{\circ} 2$ de l'AFP

34. Entretien journaliste $n^{\circ} 1$ de l'AFP

35. Cette idée revient dans au moins quatre de nos entretiens.

36. Entretien journaliste $n^{\circ} 5$ de Libération

37. ibid.

38. Certaines rédactions s'interdisent aussi les questions touchant à la vie privée.

39. Entretien journaliste $n^{\circ} 3$ de l'AFP

40. Entretien journaliste ${ }^{\circ} 3$ de Libération

41. Entretien journaliste $n^{\circ} 5$ de Libération 


\section{Entretien journaliste $n^{\circ} 3$ de Libération}

43. Qu'on ne peut pas démystifier.

44. Entretien journaliste $n^{\circ} 1$ de l'AFP. Les fact-checkeurs britanniques de Full Fact proposent ainsi d'ajouter à l'outil Facebook une catégorie "non corroboré " qui répondrait à cette situation et au problème de l'inversion de la charge de la preuve : Report on the Facebook Third Party Fact Checking programme, Jan-June 2019, p. 28. Accès : https://fullfact.org/media/uploads/tpfc-q1q2-2019.pdf consulté le 12/11/19.

45. Théorie selon laquelle les traînées blanches laissées par le passage des avions dans le ciel seraient des produits chimiques répandus par des agences gouvernementales pour des raisons cachées au public (modification du climat, régulation démographique, guerre chimique, entre autres).

46. Entretien journaliste $n^{\circ} 3$ de l'AFP

47. Entretien journaliste $n^{\circ} 1$ de l'AFP

48. Entretien journaliste $n^{\circ} 2$ de l'AFP

49. Entretien journaliste $n^{\circ} 3$ de l'AFP

50. Entretien journaliste $n^{\circ} 4$ de l'AFP

51. Entretien journaliste $n^{\circ} 1$ de l'AFP

52. Entretien journaliste $n^{\circ} 2$ de 20 Minutes et $n^{\circ} 1$ de Libération

53. Entretien journaliste $n^{\circ} 3$ de l'AFP

54. Cette pertinence est cependant très discutable : au vu des chiffres vertigineux de la communication numérique, il convient de relativiser les données brutes des interactions (Cardon, 2019).

55. Entretien journaliste ${ }^{\circ} 5$ de Libération

56. Une idée qui revient dans trois de nos entretiens

57. Entretien journaliste $n^{\circ} 3$ de l'AFP

58. Entretien journaliste $n^{\circ} 2$ de 20 Minutes

59. Entretien journaliste $n^{\circ} 4$ de Libération

60. ibid.

61. ibid.

62. Entretien journaliste $n^{\circ} 3$ de Libération

63. Entretien journaliste $n^{\circ} 1$ de Libération

64. Entretien journaliste $n^{\circ} 3$ de l'AFP

65. Entretien journaliste $n^{\circ} 4$ de Libération

66. Entretien journaliste $n^{\circ} 5$ de Libération

67. Entretien journaliste $n^{\circ} 4$ de Libération

68. Entretien journaliste $n^{\circ} 1$ de l'AFP

69. Entretien journaliste $n^{\circ} 3$ de Libération

70. Entretien journaliste $n^{\circ} 2$ de 20 Minutes et $n^{\circ} 5$ de Libération

71. Entretien journaliste $n^{\circ} 1$ de 20 Minutes. Les enquêtes sur la confiance indiquent des niveaux toujours faibles, malgré un léger regain en 2020:46\% de confiance pour la presse (33e baromètre de la confiance des Français dans les médias, en baisse de 6 points par rapport à 2018. Accès: https://www.la-croix.com/Economie/Medias/ 
Barometre-medias-pourquoi-4-Francais-10-boudent-

linformation-2020-01-15-1201072072, consulté le 02/10/20), et $28 \%$ de confiance pour les médias en général (baromètre de la confiance politique du CEVIPOF de février 2020, qui mesure également la méfiance envers les statistiques officielles. Accès : https:// www.sciencespo.fr/cevipof/fr/content/les-resultats-par-vague.html, consulté le $02 / 10 / 20$.

72. Entretien journaliste $n^{\circ} 3$ de Libération.

73. Entretien journaliste $n^{\circ} 1$ de 20 Minutes

74. Entretien journaliste $n^{\circ} 1$ de l'AFP

75. Entretien journaliste $n^{\circ} 3$ de l'AFP

76. Entretien journaliste $\mathrm{n}^{\circ} 4$ Libération

77. Entretien journaliste $n^{\circ} 3$ de l'AFP.

78. Entretien journaliste $n^{\circ} 5$ de Libération

79. ibid.

80. Entretien journaliste $n^{\circ} 2$ de l'AFP

81. Entretien journaliste $n^{\circ} 2$ de Libération

82. Entretien journaliste $n^{\circ} 1$ de l'AFP

83. Entretien journaliste $n^{\circ} 3$ de Libération

84. Entretien journaliste $n^{\circ} 2$ de 20 Minutes

85. Accès : https://ifcncodeofprinciples.poynter.org, consulté le 28/11/19.

86. La Déclaration de Grunewald sur l'éducation aux médias a été adoptée en janvier 1982 dans le cadre de l'UNESCO. Accès: http://www.unesco.org/education/pdf/ MEDIA_F.PDF, consulté le 30/11/19; en France, l'éducation aux médias et à l'information (EMI), inscrite dans le "parcours citoyen » des élèves, est fortement promue dans l'enseignement secondaire, plus particulièrement depuis 2015. Elle est notamment mise en valeur dans la circulaire de rentrée 2017 (circulaire n 2017-045 du 9-3-2017, MENESR - DGESCO. Accès: https://www.education.gouv.fr/pid285/ bulletin_officiel.html?cid_bo=113978, consulté le 30/11/19).

87. Entretien journaliste $n^{\circ} 3$ de l'AFP

88. ibid. Il est par ailleurs intéressant de noter que les trois cellules étudiées ont traité ce sujet. Voir Jacques Pezet, "Les lions noirs existent-ils?", CheckNews/Libération, 17/09/18. Accès : https://www.liberation.fr/checknews/2018/09/17/les-lions-noirsexistent-ils_1679243, consulté le 26/12/2020. Fake Off/20 Minutes, "Lions noirs: des images spectaculaires, mais manipulées ", 07/09/19. Accès : https://www.20minutes.fr/ planete/2598575-20190907-lions-noirs-images-spectaculaires-manipulees, consulté le 26/12/2020. Factuel/AFP, 08/10/19, « Non, les lions noirs n'existent pas, ces photos ont été truquées ». Accès : https://factuel.afp.com/non-les-lions-noirs-nexistent-pas-cesphotos-ont-ete-truquees, consulté le 26/12/2020.

89. Entretien journaliste $n^{\circ} 3$ de Libération

90. Entretien journaliste $n^{\circ} 4$ de Libération et $n^{\circ} 1$ de l'AFP

91. Entretien journaliste $n^{\circ} 1$ de l'AFP

92. Entretien journaliste $n^{\circ} 3$ de l'AFP

93. Entretien journaliste ${ }^{\circ} 1$ de Libération 
94. Entretiens journalistes $n^{\circ} 2$ de 20 Minutes et $n^{\circ} 5$ de Libération

95. Entretien journaliste $n^{\circ} 1$ de l'AFP

96. Entretien journaliste $n^{\circ} 2$ de l'AFP

97. Entretien journaliste $n^{\circ} 3$ de l'AFP

98. $i$ bid.

99. Entretien journaliste $n^{\circ} 5$ de Libération

100. Entretien journalistes $n^{\circ} 2$ et 3 de l'AFP

101. Entretien journaliste $n^{\circ} 1$ de l'AFP

102. Entretien journaliste $n^{\circ} 1$ de Libération

103. Entretiens journalistes $n^{\circ} 2$ et 5 de Libération

104. Entretiens journalistes $n^{\circ} 3$ et 4 de Libération

105. Entretien journaliste $n^{\circ} 1$ de l'AFP

106. Entretien journaliste $n^{\circ} 2$ de 20 Minutes

107. Entretien journaliste $n^{\circ} 3$ de l'AFP

108. Entretien journaliste $n^{\circ} 1$ de l'AFP

109. Entretien journaliste $n^{\circ} 1$ de Libération

110. Entretien journaliste $n^{\circ} 5$ de Libération

111. Entretien journaliste $n^{\circ} 1$ de l'AFP

112. Entretien journaliste $n^{\circ} 3$ de Libération

113. Entretien journaliste $n^{\circ} 5$ de Libération

114. Entretiens journalistes $n^{\circ} 1,2$ et 3 de Libération

115. Entretien journaliste $n^{\circ} 3$ de l'AFP

116. ibid.

117. Entretien journaliste $n^{\circ} 4$ de Libération

118. Entretien journaliste $n^{\circ} 1$ de 20 Minutes

119. Entretien journaliste $n^{\circ} 4$ de Libération

120. Entretien journaliste $n^{\circ} 5$ de Libération

121. Entretien journaliste $n^{\circ} 1$ de 20 Minutes

122. Entretien journaliste $n^{\circ} 3$ de Libération

123. Entretien journaliste $n^{\circ} 5$ de Libération

\section{RÉSUMÉS}

Le propos de cet article est d'analyser le rapport des principaux services français de factchecking aux «théories du complot » et à la façon par laquelle les stratégies de contournement de cette catégorie repoussoir contribuent à définir les «théories du complot » comme déviances informationnelles et à réaffirmer la légitimité du travail de fabrication des « faits journalistiques 
" dans une démocratie. Après avoir analysé le discours des fact-checkeurs à propos du « complotisme ", nous examinons comment cette catégorie opère dans la mise en œuvre des critères de sélection et de traitement des sujets à vérifier. Nous montrons ensuite comment l'un des enjeux de ce nouveau produit journalistique est de rétablir un lien de confiance auprès de publics vulnérables aux " lectures complotistes de l'actualité », d'une part en s'investissant d'une mission pédagogique et d'autre part en déployant des efforts de communication directe avec les lecteurs.

The purpose of this article is to analyze the relationship of the main French fact-checking services to "conspiracy theories" and how the circumvention strategies of this repulsive category helps to define "conspiracy theories" as informational deviances and to reaffirm the legitimacy of the work of elaboration of "journalistic facts" in a democracy. After having analyzed the discourse of fact-checkers about "conspiracy theories", we examine how this category operates in the implementation of the criteria for selecting and treating topics to be verified. We then show how one of the challenges of this new journalistic product is to re-establish a bond of trust with audiences vulnerable to "conspiratorial interpretations of the news", on the one hand by investing in an educational mission and on the other hand by deploying direct communication efforts with readers.

INDEX

Mots-clés : journalisme, complotisme, infox, vérification factuelle, démocratie

Keywords : journalism, conspiracy, fake-news, fact checking, democracy

\section{AUTEURS}

ELSA JAUBERT

UNICAEN, ERLIS EA 4254

VASSILI RIVRON

UNICAEN, CERREV EA 3918 